\title{
O AXIOMA DA TRANSITIVIDADE É SUPÉRFLUO?*
}

\author{
Juan Hersztajn Moldau**
}

\section{Resumo}

Este ar tigo trata de uma discussão acerca da necessidade de uma condição de transitividade na teoria da demanda. As tentativas melhor conhecidas para descartar a hipótese de transitividade empregam uma condição de convexidade. Neste trabalho demonstramos que a condição de convexidade implica na propriedade de transitividade sobre a linha reta. Apresentamos em seguida uma prova simples de existência de uma correspondência de demanda no espaço bidimensional que faz uso da propriedade de transitividade que está implícita na condição de convexidade.

\begin{abstract}
This paper is concerned with a discussion about the necessity of a transitivity axiom in choice theory. The best known attempts to dismiss the transitivity property employ the convexity condition. In this article we show that the convexity condition implies transitivity in all linear directions. We provide then a simple proof of the existence of a demand correspondence in a two-dimensional commodity space without imposing an explicit transitivity condition. A brief discussion on the meaning of the transitivity property that is implicit in the convexity condition concludes the paper.
\end{abstract}

\section{Introdução.}

Os modelos de escolha via de regra impõem a hipótese de transitividade com referência à relação de preferência. Este postulado ć essencial para definição de uma pré-ordenação de alternativas a qual por sua vez, ć considerada necessária para a obtenção de uma correspondência de demanda.

$\mathrm{O}$ axioma de transitividade tem sido debatido na literatura tendo em vista dúvidas levantadas quanto ao seu realismo (veja por exemplo, May, 1954 e Tversky, 1969). A insatisfação com a restrição associada a este postulado têm estimulado tentativas dirigidas ao seu relaxamento. Um avanço importante nesta direção foi proporcionado por Sonnenschein (1971), o qual demonstrou a existência de uma

\footnotetext{
*Devo um agradecimento especial a dois pareceristas desta revista por seus comentários e valiosas sugestões.

**O autor é professor da Fea/USP

\begin{tabular}{llll}
\hline R. de Econometria & Rio de Janeiro & v. XI, $\mathrm{n}^{\circ} 1, \mathrm{p} .93-98$ & abril 1991 \\
\hline
\end{tabular}
}


função de demanda sem a necessidade de imposição do axioma de transitividade - a hipótese de convexidade seria suficiente. Outros estudos têm confirmado este resultado (veja Shafer 1974, Kihlstrom, Mas Colell e Sonnenschein, 1976 e Kim e Richter, 1986).

Neste artigo fazemos a conjectura de que a hipótese de convexidade implica numa condição de transitividade, muito embora csta seja relativamente mais branda que a usual. Isto implica que a teoria da demanda requer necessariamente pelo menos alguma forma de transitividade a qual poderia então ser proporcionada pela propriedade de convexidade. Neste trabalho mostramos que no espaço bidimensional a existência de uma correspondência (função) de demanda pode ser demonstrada pelos procedimentos usuais sem a imposição explícita da propriedade de transitividade. Bastaria para isso estipular uma condição de convexidade fraca (estrita) que teria então o efeito de proporcionar um grau suficiente de transitividade.

A sequência deste artigo está organizada como se segue: Na seção 2 ć feito um exame das implicações da condição de convexidade, na ausência de uma hipótese de transitividade, com relação a um modelo usual de escolha. Na seção 3 são apresentadas as principais conclusões do trabalho.

2. A convexidade como condiçāo substitutiva da de transitividade.

Nesta seção iniciaremos aplicando a definição usual de convexidade para mostrar que esta implica na satisfação da propriedade de transitividade com referência aos elementos localizados sobre uma linha reta - inversamente, a falta de transitividade relativamente a estes elementos implica na ausência da condição de convexidade.

Considere o conjunto de escolha $X \subseteq \mathrm{R}^{n}$. A variável $x$ representa um elemento de $X$ sendo um vetor composto por até $n$ componentes, $x_{1}, \ldots x_{j}, \ldots x_{n}$; cada qual representando a quantidade de um bem.

Assumiremos que $x_{j}$, para $j=1, \cdots n$, ć um número cardinalmente mensurável e perfeitamente divisívcl. A relação de preferência fraca $R$, representa o conceito primitivo básico do modelo.

Baseados em $R$ podemos definir as relações de preferência estrita (P) e de indiferença (I); 


$$
\begin{aligned}
& \forall x, y \in X: \quad x P y \Longleftrightarrow x R y \text { с } \neg y R x \\
& x I y \Longleftrightarrow x R y \text { с } y R x \text {. }
\end{aligned}
$$

A análise aqui desenvolvida estará baseada no seguinte sistema de axiomas:

$A_{1} \cdot \forall x, y \in X: x R y$ ou $y R x$ (comparabilidade).

$A_{2}(a) \cdot \forall x, y \in X: x P y \rightarrow z P y ; z=t x+(1-t) y, 0<t<1$ (convexidade fraca).

$A_{2}(b) \cdot \forall x, y \in X: x I y \rightarrow z p x, z P y ; z=t x+(1-t) y, 0<t<1$ (convexidade estrita).

$A_{3}$. Considere $x=\left(x_{1}, \cdots, x_{n}\right)$ e $y=\left(y_{1} \cdots y_{n}\right) ; x, y \in X$. Se $x_{s}>y_{s}$ e $x_{r} \geq y_{r}$, para $r \neq s$, então $x R y, \forall s \leq n$ e $x P y$ para algum $s \leq n$. (monotonicidade).

$A_{4} \cdot \forall x^{\prime} \in X:$ Os conjuntos $\left\{x \in X / x R x^{\prime}\right\}$ e $\left\{x \in X / x^{\prime} R x\right\}$ são fechados em $X$ (continuidade).

Denominaremos por ciclo $P$ qualquer padrão de escolha intransitiva do tipo $x P y P z P x$. Demonstraremos a seguir que $A_{2}(a)$ implica que pontos sobre a linha reta satisfazem a propriedade de transitividade:

Lema 1. Considerc a linha reta $x, y$ para $x \neq y \mathrm{~cm} X$. Dado $A_{2}(a)$ a propriedadc de transitividade é satisfeita por todos os pontos sobre a linha reta $x, y$.

Prova. Consideremos todos os ciclos $P$ correspondentes a quaisquer combinaçöes $x, y, z$ sobre a linha reta $x, y$ onde $z=t x+(1-t) y$ para $t \in \mathbf{R}$ :

$x P y, y P z, z P x ; y P x, x P z, z P y ; x P z, z P y, y P x ; z P x, x P y, y P z ; y P z$, $z \mathrm{Px}, \mathrm{xPy} ; \mathrm{zPy}, \mathrm{yPx}, \mathrm{xPz}$.

$A_{2}(a)$ implica que excluída a relação $x I y$ temos necessariamente uma das seguintes combinações de relações de preferência: $x P y$ e $z P y$ ou $y P x$ e $z P x$. Portanto todos os ciclos intransitivos de preferência envolvando $x, y$ e $z$ são excluídos se $A_{2}(a)$ ć imposto sobre $R$ (pode-se verificar facilmente que combinações do tipo $x P y P z R x$ são também excluídas). Verificamos também que reciprocamente, a existência de ciclos $P$ invalida a condição de convexidade. 
Corolário 1. Considerc a linha reta $x, y$ para $x \neq y \mathrm{~cm} X$. Dados $A_{2}(b) e A_{4}$ a propricdade de transitividade ć satisfeita por todos os pontos sobrc a linha reta $x, y$.

Prova. Dado $A_{4}, A_{2}(b)$ implica $A_{2}(a)$ (Cf. Debreu, 1959, p.61). Portanto podemos aplicar dirctamente o Lema 1.

A partir do Lema 1 c do Corolário 1 podemos demonstrar a existência de uma correspondência ou função de demanda no espaço bidimensional. Nesta demonstração emprega-sc um método conhccido (vcja por excmplo, Uzawa, 1971) o qual ć bascado no cumprimento da propricdade de transitividade.

Considere a restrição orçamentária definida pela correspondência $\psi(p, M)=\{x \in X / p x \leq M\}$, onde o sistema de preços $p=\left(p_{1}, p_{2}\right)$ с $M$ corresponde à renda do agentc. Scja $B=\{x \in X / p x=M\}$ a frontcira de $\psi(p, M)$.

Scja $S=\left\{(p, M) \in \mathbf{R}^{3}\right\}$ para $M, p>0$. A correspondência de demanda $\phi(p, M)$, de $S$ para $X$, ć definida pclo subconjunto de elementos maximais $\mathrm{cm} \psi(p, M)$ segundo $R$.

Teorema 1. Dados $A_{1}, A_{2}(a), A_{3}$ c $A_{4}$ cxistc uma correspondência dc demanda $\phi(p, M)$, dc $S$ para $X \subseteq \mathbf{R}^{2}$.

Prova. $A_{3}$ implica que o subconjunto de clementos maximais de $\psi(p, M)$ segundo $R$ pertence a $B$. Portanto a tarefa de determinar $\phi(p, M)$ se reduz à de encontrar o subconjunto de clementos maximais em $B$ segundo $R$. Como todos os clementos situados no interior de $\psi(p, M)$ estão excluídos como possíveis componentes de $\phi(p, M)$ segue-se que possívcis ciclos $P$ obscrvados com relação a estes elementos são irrelevantes para cfeito de determinação da correspondência de demanda. Scrá suficiente a definição de uma pré-ordenação completa com relação apenas aos clementos de cada $B$. Bastará então que scja satisfeita a propricdade de transitividade com referência a estes clementos. Conforme o Lema 1 esta condição ć satisfeita através da imposição de $A_{2}(a)$. A prova da existência de um subconjunto não vazio de elementos maximais segundo $R \mathrm{em} B$ pode ser obtida diretamente a partir da prova disponível para o caso de uma restrição orçamentária convencional (vcja por excmplo, Uzawa, 1971).

Deveremos mostrar inicialmente que existe $x^{0} \mathrm{em} B$ tal que 


$$
x^{0} R x, \forall x \in B
$$

Considcrando qualquer $x \in B$, defina o conjunto $C_{x}=\{y: y \in$ $B, y R x\}$. Para qualquer número finito de combinações $x_{1} \ldots x_{r} ; C_{x^{1}} \cap$ $\cdots \cap C_{x^{r}} \neq \emptyset$. Este resultado ć consequência de $B$ scr compacto c $R$ transitiva $\mathrm{cm} B$. Por $A_{4}, C_{x}$ ć fechado $\mathrm{cm} B$. Portanto $\bigcap_{x} \neq \emptyset$ (dado que $B$ ć compacto c $C_{x}$ fechado $\mathrm{cm} B$ (vcja Uzawa, 1971, p.23)). Considere qualquer $x^{0} \mathrm{~cm} \bigcap_{x \in B} C_{x}$. Dada a definição de $C_{x}$, temos $x^{0} R x$, para todo $x \in B$ c portanto fica cstabclecida a cxistência de uma correspondência de demanda de $S$ para $X$.

Corolário 2. Dados $A_{1}, A_{2}(b), A_{3}$ c $A_{4}$ cxistc uma função dc dcmanda dc $S$ para $X \subseteq \mathbb{R}^{2}$.

Prova. A prova ć por contradição. Assuma que $x^{0}$ c $x$ cstcjam contidos cm $\bigcap_{x \in B} C_{x}$. Dado $A_{2}(b)$ c sendo $B$ convexo existc $x^{\prime}=t x^{0}+(1-t) x$ para $0<t<1 \mathrm{~cm} B$ c tal que $x^{\prime} P x^{0}$. Isto contradiz (1). Portanto há um único clemento maximal $\mathrm{cm} B$ segundo $R$ o que prova a existência de uma função de demanda de $S$ para $X$.

\section{Conclusāo.}

O importante artigo de Sonnenschein de 1971, c trabalhos posteriores sugerem que o axioma de transitividade ć desnecessário para se demonstrar a existência de uma correspondência ou função de demanda. Bastaria que o modelo de escolha proposto incluísse uma hipótese de convexidade.

Neste artigo argumentamos que a determinação de uma função ou correspondência de demanda requer necessariamente, uma forma, mesmo que mais branda de transitividade. Isto significa que a propricdade de transitividade deverá ser imposta pelo menos cm relação a um subconjunto menor de combinaçõcs de alternativas. A imposição de uma hipótese de convexidade garantiria então a satisfação desta condição menos restritiva de transitividade. Portanto não ć válida a assertiva de que a condição de transitividade scja totalmente 
supćrflua na tcoria da demanda. Em outras palavras, esta tcoria não admitc todas as formas de comportamento intransitivo. Podemos, entretanto accitar a conclusão de que a condição de transitividade implícita na hipótese de convexidade admite a ocorrência de ciclos $P$ com relação a detcrminadas combinações de alternativas os quais seriam irrelevantes para efcito de determinação de correspondências de demanda.

\section{Referências}

Debrcu, G. Theory of Value: An Axiomatic Analysis of Economic Equilibrium. New Haven \& London, Yalc University Press, 1959. Kihlstrom, R. Mas Colcll, A. c Sonnenschein, H. The Demand Theory of the Weak Axiom of Revealed Preference. Econometrica, 44:971-978, 1976.

Kim, T. and Richter, M. Non Transitive-Non Total Consumer Theory. Journal of Economic Theory, 38: 324-363, 1986.

May, K.C. Intransitivity, Utility and the Aggregation of Preference Pattcrns. Econometrica, 22:1-13, 1954.

Shafer, W.J.. The Non-Transitive Consumer. Econometrica, 42: 913-919, 1974.

Sonnenschein, H. Demand Theory Without Transitive Preferences With Application to the Thcory of Compctitive Equilibrium. in Preferences Utility and Demand, Chipman, J,S. et al. eds. New York: Harcourt Bracc, 215-223, 1971.

Tversky, A. Intransitivity of Preferences. Psychological Review, 76: 31-48, 1969.

Uzawa,H. Preference and Rational Choice in the Theory of Consumption. in Preferences Utility and Demand,Chipman, J.S. et al. eds. New York, Harcourt Brace, 7-28, 1971. 